Article

\title{
Ensino de Meteorologia e Climatologia na Formação Continuada do Agente de Proteção e Defesa Civil: Estudo de Caso na Região Semiárida do Nordeste do Brasil
}

\author{
Emerson Mariano da Silva $^{1}$ (D), Joel de Abreu Nobre ${ }^{1,2}$, Wellington Antonio Barbosa ${ }^{3}$ \\ ${ }^{1}$ Mestrado Profissional em Climatologia e Aplicações nos Países da CPLP e África, Universidade \\ Estadual do Ceará, Fortaleza, CE, Brasil. \\ ${ }^{2}$ Corpo de Bombeiros Militar do Ceará, Fortaleza, CE, Brasil. \\ ${ }^{3}$ Agência Executiva de Gestão das Águas do Estado da Paraíba, Campina Grande, PB, Brasil.
}

Recebido em: 18 de Fevereiro de 2021 - Aceito em: 14 de Abril de 2021

\begin{abstract}
Resumo
Apresenta-se uma investigação sobre o ensino de Meteorologia na formação e nos cursos de capacitação (formação continuada) ofertados aos agentes de proteção e defesa civil que atuam em estados da Região Semiárida do Nordeste do Brasil (RSANEB). Foi usado um questionário eletrônico com quinze perguntas referentes aos conhecimentos dos participantes sobre dos fenômenos meteorológicos que modulam o clima da RSANEB, sobre os desastres associados a eventos extremos climáticos (secas e enchentes) e sobre a formação continuada dos agentes de proteção e defesa civil que atuam na região. Os resultados mostram que para serem capazes de compreender a dinâmica desses fenômenos e, com isso, desenvolver planos com ações (preventivas, responsivas e de recuperação) para mitigar os efeitos dos impactos da variabilidade climática observados em suas comunidades, o processo de formação dos agentes de proteção e defesa civil que atuam na RSANEB necessita passar por reformulações, incluindo e/ou reforçado a carga horária da ministração de conteúdos sobre os conhecimentos básicos dos fenômenos meteorológicos que modulam o clima e podem causar desastres (secas ou enchentes) na região.
\end{abstract}

Palavras-chave: meteorologia, ensino de climatologia, formação em defesa civil.

\section{Teaching Meteorology and Climatology in the Continuing Education of the Civil Defense and Protection Agent: Case Study in the Semi-Arid Region of Northeast Brazil}

\begin{abstract}
An investigation is presented on the teaching of Meteorology in training and in training courses (continuing education) offered to civil protection and defense agents working in states in the Semi-Arid Region of Northeast Brazil (RSANEB). An electronic questionnaire was used with fifteen questions regarding the participants' knowledge about the meteorological phenomena that modulate the RSANEB climate, about the disasters associated with extreme climatic events (droughts and floods), and about the continued training of civil defense and defense agents operating in the region. The results show that in order to be able to understand the dynamics of these phenomena and, with that, develop plans with actions (preventive, responsive and recovery) to mitigate the effects of the impacts of climatic variability observed in their communities, the process of training agents of protection and civil defense that work in RSANEB needs to undergo reformulations, including and/or reinforced the workload of teaching contents on the basic knowledge of meteorological phenomena that modulate the climate and can cause disasters (droughts or floods) in the region.
\end{abstract}

Keywords: meteorology, climatology teaching, continuing education of the civil defense.

Autor de correspondência: Emerson Mariano da Silva. E-mail: emerson.mariano@uece.br. 


\section{Introdução}

A Região Semiárida do Nordeste do Brasil (RSANEB) possui uma área de $1.127 .953 \mathrm{~km}^{2}$, contendo 1.262 municípios. A recente delimitação da RSANEB levou em consideração os seguintes critérios: precipitação pluviométrica média anual inferior a 800 milímetros, índice de aridez de até 0,50 (cálculo baseado no método de Thornthwaite) e o percentual diário de déficit hídrico igual ou superior a $60 \%$, considerando todos os dias do ano (SUDENE, 2017).

Segundo relatórios do Intergovernamental Painel on Climate Change (IPCC, 2007) a RSANEB é uma das regiões do país mais afetadas pelas mudanças climáticas, uma vez que, além dos cenários de aumento de temperatura do ar, a região apresenta tendência de se tornar mais árida, com possibilidade de aumento da frequência de ocorrência de secas, reduzindo, assim, a disponibilidade de recursos hídricos na região.

Segundo Marengo (2006) a RSANEB é afetada por grandes secas ou grandes cheias que acontecem de 18 a 20 anos a cada 100 anos. O regime pluviométrico delimita duas estações bem distintas: a estação das chuvas (primeiro semestre), com duração de três a cinco meses, e a estação sem ou com pouco ocorrência de chuvas (seca) com duração de sete a nove meses por ano.

O estudo comportamental do regime de chuvas na RSANEB tem uma importância significativa para o povo dessa região, pois à irregularidade apresentada em escalas de tempo e espaço trazem consequências de ordem econômica, social e cultural.

Menciona-se, ainda, que a variabilidade do clima observado na RSANEB é modulada por padrões atmosféricos e oceânicos de grande escala sobre os Oceanos Pacífico e Atlântico Tropicais (Rao e Hada, 1990; Marengo, 2006). Nesse contexto, para o entendimento da variabilidade climática observada na RSANEB faz-se necessário conhecer e compreender a dinâmica dos diversos sistemas atmosférico-oceânicos que atuam nessa modulação: El Niño, La Niña, Zona de Convergência Intertropical ZCIT, Distúrbios Ondulatórios de Leste - DOLs, Vórtice Ciclônico de Altos Níveis - VCAN (Kousky e Gan, 1981; Rasmusson e Carpenter, 1982; Uvo, 1989; Chou, 1990; Ferreira e Mello, 2005; Marengo, 2009; Alves, 2012).

Cantero (2015) e Giddens (2009) concordam que as mudanças no clima e seus impactos, em determinada região, constituem uma questão fundamental rumo à novas ações, estratégias de mitigação e adaptação e um dos maiores desafios a serem enfrentados pelas sociedades atual e futura, pois tais alterações afetam, principalmente, os mais pobres, que representam uma grande parcela da sociedade vivendo em situação de vulnerabilidade socioeconômica, expostos a ocorrência de eventos extremos como chuvas torrenciais, que causam inundações, alagamentos e desabamentos, e secas que causam a escassez de alimento nessas comunidades.
Diante deste quadro que se apresenta, ressalta-se a importância e a necessidade do ensino de Meteorologia, com ênfase nos conceitos e na dinâmica dos fenômenos meteorológicos que modulam a variabilidade do clima na RSANEB, na formação dos agentes de proteção e defesa civil da região, para que sejam capazes de compreender a dinâmica desses fenômenos e, com isso, desenvolver planos com ações (preventivas, responsivas e de recuperação) para mitigar os efeitos dos impactos da variabilidade climática observados em suas comunidades.

Assim, o objetivo deste estudo é diagnosticar os conhecimentos básicos sobre os fenômenos meteorológicos que modulam o clima da RSANEB e o processo de formação continuada dos agentes de proteção e defesa civil que atuam na região.

\subsection{Processo de formação continuada do Agente de Proteção e Defesa Civil}

O início das atividades de defesa civil no país e de sua institucionalização, dar-se através da edição do Decreto Lei ${ }^{\circ} 4.098$ de 1942, assinado pelo presidente brasileiro Getúlio Vargas, que criou o Serviço de Defesa Passiva Antiaérea, associado ao Ministério da Aeronáutica (Carchedi, 2006). Assim, a defesa civil surge como um órgão responsável pela prestação de serviços preventivos, de socorro e minimização dos impactos causados pelos desastres (naturais, tecnológicos ou causados pela ação humana).

A mais recente norma que trata do conceito de defesa civil no país, a instrução normativa $n^{\circ} 02 / 2016$ do Ministério da Integração Nacional (MI), incorporou o termo "proteção", no sentido de destacar o caráter integral e preventivo das ações e traz a seguinte definição: "Conjunto de ações de prevenção, mitigação, preparação, resposta e recuperação destinadas a evitar desastres e minimizar seus impactos sobre a população e a promover o retorno à normalidade social, econômica ou ambiental" (BRASIL, 2016 e 2017).

A Política Nacional de Proteção e Defesa Civil (PNPDEC), estabelecida por meio da Lei ${ }^{\circ} 12.608$ de 10 de abril de 2012, é o marco doutrinário da proteção e defesa civil no país, expresso pelas diretrizes e objetivos instituídos na política e que devem ser seguidos por todos os membros do Sistema Nacional de Proteção e Defesa Civil (SINPDEC).

A PNPDEC abrange as ações de prevenção, mitigação, preparação, resposta e recuperação, representa um conjunto dessas ações em um processo integrado, permanente e interdependente, em busca da gestão integrada em proteção e defesa civil: "[...] às políticas de ordenamento territorial, desenvolvimento urbano, saúde, meio ambiente, mudanças climáticas, gestão de recursos hídricos, geologia, infraestrutura, educação, ciência e tecnologia e às demais políticas setoriais, tendo em vista a promoção do desenvolvimento sustentável” (BRASIL, 2012). 
Nesse contexto, encontra-se o agente de defesa e proteção civil e sua formação, que segundo Delors (2003) é rica em saberes e muito complexa, uma vez que objetiva formar profissionais com conhecimento das normas técnicas, teóricos e práticos, esses últimos que atendam as necessidades de atender as ocorrências em campo. Portanto, segundo o autor, faz-se necessário que o profissional de proteção e defesa civil participe de um processo de formação continuada.

$\mathrm{O}$ autor em suas reflexões sobre o processo de formação continuada dos agentes de proteção e defesa civil corrobora com as ideias de Nóvoa (1991) e de Freire (1996), ressaltando que esse processo deve propor novas metodologias e provocar discussões teóricas e vivências práticas, com o objetivo de contribuir para as mudanças que se fazem necessárias na atuação em sociedade.

Assim, atenta-se para a importante missão do processo de formação continuada dos profissionais de proteção e defesa civil, especificamente aos que se encontram nos estados contidos na RSANEB, que vivem e atuam diretamente em regiões de grande vulnerabilidade socioeconômica, suscetíveis aos efeitos associados as variabilidades (espacial e temporal) do clima e que observam, historicamente, desafios associados as ocorrências de secas ou enchentes.

\section{Metodologia e Dados}

Para a coleta de informações que objetivou esse estudo foi usado um questionário através de um formulário eletrônico, contendo 15 (quinze) perguntas relacionadas aos temas: conhecimento dos fenômenos meteorológicos que modulam o clima da RSANEB e sobre a formação continuada dos agentes de proteção e defesa civil que atuam na região. Os formulários eletrônicos permitem que o usuário obtenha e organize (gratuitamente) informações, as respostas obtidas são armazenadas em planilhas para futura visualização e análises.

Segundo Fachin (2001) "esse tipo de instrumento de coleta de dados consiste num elenco de questões que são apreciadas e submetidas a certo número de pessoas com o intuito de obter respostas para a coleta de informações". Além disso, para a autora o número de questões deve ser reduzido, ter redação simples, completa e clara com o intuito de prender a atenção do participante.

De acordo com Gil (2008), o questionário pode ser definido como uma técnica de investigação "[...] composta por um número mais ou menos elevado de questões apresentadas por escrito às pessoas, tendo por objetivo o conhecimento de opiniões, crenças, sentimentos, interesses, expectativas, situações vivenciadas e etc".

A utilização de questionários apresenta uma série de vantagens e de limitações, Marconi e Lakatos (2005) destacam as seguintes vantagens: economia de tempo e longos deslocamentos, obtenção de grande número de informações, atinge maior número de pessoas simultaneamente, liberdade nas respostas em razão do anonimato. Por outro lado, as limitações mais evidentes são, as possíveis: pequena percentagem de retorno e devolução tardia que pode prejudicar o calendário de sua utilização.

Assim, o instrumento de coleta usado nesse estudo foi distribuído por e-mail as Coordenadorias de Proteção e Defesa Civil (CEDECs) dos estados que contém municípios na RASNEB, agregando-se uma carta de apresentação da pesquisa. O questionário foi disponibilizado para as respostas dos agentes por 15 (quinze) dias, tempo suficiente para que os coordenadores tivessem repassassem para os agentes estaduais e fazer uma articulação com as Coordenadorias Municipais de Proteção e Defesa Civil (COMPEDCs) em cada estado, com isso foram obtidas 141 (cento e quarenta e uma) respostas de agentes que atuam nos municípios dos estados do Ceará, do Piauí, do Rio Grande do Norte, de Pernambuco e de Alagoas.

A participação dos estados da Paraíba, Bahia e de Sergipe não ocorreram de forma satisfatória devido à falta de retorno das informações, por outro lado, foi descartada a participação do estado do Maranhão por possuir apenas dois municípios na RSANEB.

\section{Resultados e Discussões}

Os resultados apresentados a seguir representam uma compilação das respostas obtidas com o envio dos questionários eletrônicos, assim, serão apresentados cinco dos principais pontos investigados.

A Fig. 1 mostra os resultados da investigação sobre o conhecimento dos fenômenos meteorológicos que modulam o clima da RSANEB (autodeclaração). Observa-se que as respostas obtidas indicam o desconhecimento da complexidade dos conteúdos associados as variabilidades climáticas observadas na RSANEB e que causam secas e enchentes na região. Apenas $20 \%$ das respostas são afirmativas, em adição, encontra-se que $45,3 \%$ do total afirmam dominar esses conteúdos e $34,5 \%$ não tiveram segurança em declarar sua resposta.

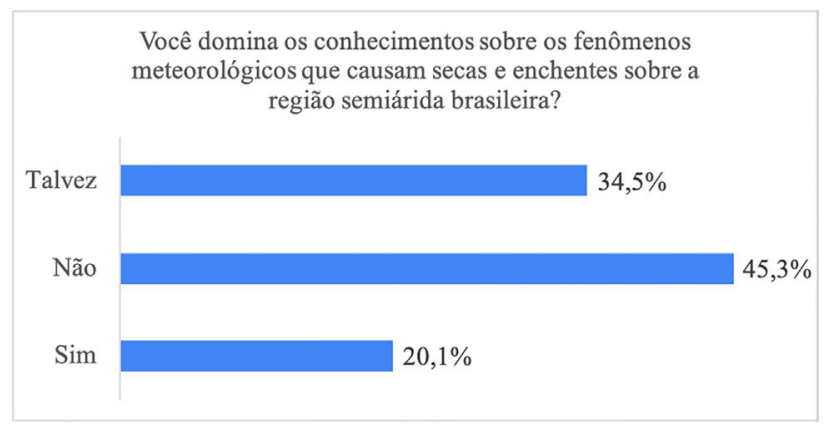

Figura 1 - Resultados obtidos: autodeclaração de conhecimentos sobre os fenômenos meteorológicos que atuam na RSANEB. Fonte: Banco de respostas da pesquisa (2020). 
$\mathrm{Na}$ sequência, mostra-se (Fig. 2) os resultados da investigação sobre nível de conhecimento dos agentes de proteção e defesa civil que atuam na RSANEB sobre os principais fenômenos meteorológicos que influenciam a ocorrência de secas e enchentes (desastres naturais) na região RSANEB. Observa-se que apenas $13,4 \%$ das respostas foram avaliadas como satisfatórias, respostas em que os agentes conseguiram associar fenômenos e sistemas meteorológicos, como a ocorrência El Niño e La Niña, bem como da Zona de Convergência Intertropical (ZCIT), com a ocorrência de chuvas intensas ou de secas na RSANEB.

Ressalta-se que a maioria dos participantes da pesquisa mostram não ter conhecimentos dessa natureza, ou seja, foram $86,5 \%$ das respostas avaliadas como insatisfatórias, respostas que não expressam minimamente a relação entre os fenômenos e sistemas meteorológicos com a ocorrência das chuvas e secas observadas na RSANEB.

Obtiveram-se respostas diversas (insatisfatórias) que expressam causas e consequências das ocorrências de secas e chuvas intensas na RSANEB, tais como a associação da desertificação e do desmatamento florestal, das ilhas urbanas de calor, das consequências das mudanças climáticas, entre outras, na tentativa de explicar o que foi solicitado como resposta da questão. Assim, constata-se que a maioria dos agentes de proteção e de defesa civil, participantes da pesquisa $(86,5 \%)$, não possuem conhecimentos sobre os fenômenos meteorológicos que modulam a variabilidade climática observada na RSANEB.

A expectativa do conhecimento dos participantes sobre os alertas meteorológicos é mostrada na Fig. 3. Os resultados obtidos apontam que 78,3\% afirmam (autodeclaração) ter conhecimentos sobre os principais sistemas de alerta de monitoramento emitidos pelos centros operacionais de meteorologia, outros $21,2 \%$ afirmam que os cursos de defesa civil não abordam temas relacionados aos alertas.

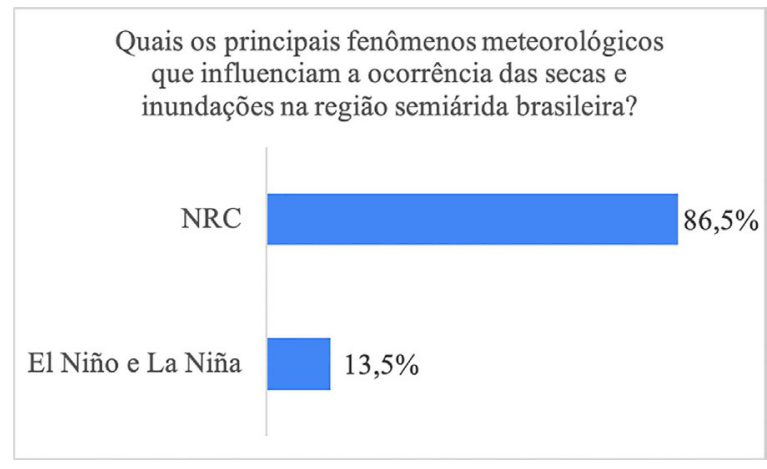

Figura 2 - Expectativa sobre o conhecimento dos fenômenos e sistemas meteorológicos que causam secas e enchentes na RSANEB (*NRC - Não responderam corretamente). Fonte: Banco de respostas da pesquisa (2020).

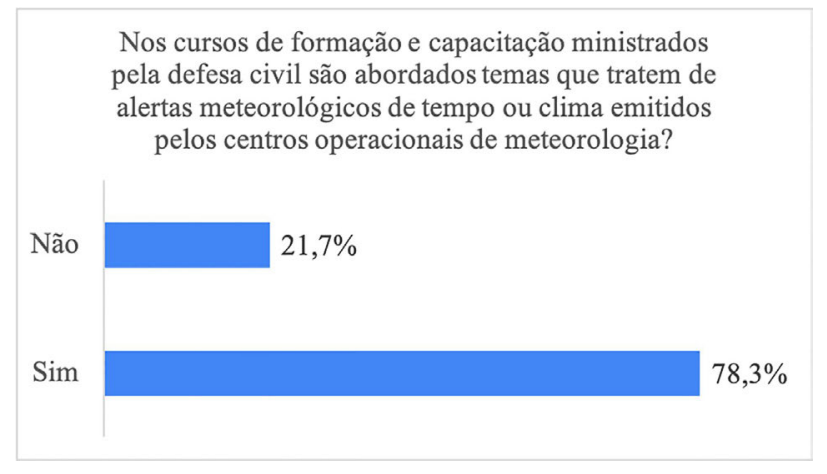

Figura 3 - Expectativa sobre o conhecimento de alertas meteorológicos dos agentes de proteção e defesa civil da RSANEB. Fonte: Banco de respostas da pesquisa (2020).

Com o quadro das respostas obtidas na pesquisa (Figs. 1, 2 e 3), mais as afirmativas de 76,1\% dos participantes sobre a oferta dos cursos de formação continuada (anual ou semestralmente, e de forma presencial), é possível levantar a hipótese de que os cursos de formação e capacitação (formação continuada) ofertados aos agentes de proteção e defesa civil que atuam na RSANEB necessitam agregar conhecimentos sobre os sistemas meteorológicos que modulam o clima, sobre a climatologia das chuvas e ocorrência de fenômenos extremos e desastres naturais associados a esses eventos observados na região.

Nesse contexto, dentre as perguntas do questionário eletrônico, procurou-se investigar a opinião dos participantes sobre a satisfação em relação aos conteúdos ministrados nos cursos de formação continuada ofertados, perguntou-se se esses conteúdos são suficientes para a formação dos agentes de proteção e defesa civil, especificamente, para o enfrentamento dos desastres naturais associados as variabilidades climáticas observadas na RSANEB.

Os resultados mostram que $54,1 \%$ afirmam que os conteúdos ministrados não são suficientes para formar o agente de proteção e defesa civil para o enfrentamento aos desastres naturais associados as variabilidades climáticas e $45,9 \%$ consideram os assuntos suficientes para essa formação em específico (Fig. 4).

Ressalta-se que, mesmo obtendo um percentual relevante entre os participantes que afirmam ter conteúdos suficientes para tal enfrentamento, as respostas sobre o conhecimento teórico (Figs. 1, 2 e 3) não confirmam essa situação. Assim, tem-se a hipótese que os participantes da pesquisa talvez tenham confundido a pergunta e expressado suas opiniões sobre os conhecimentos práticos (vivência) em campo na atuação em desastres dessa natureza.

Sobre as expectativas dos participantes em relação a importância dos conteúdos relacionados ao enfrentamento dos desastres naturais associados as variabilidades climáticas e/ou mudanças climáticas na RSANEB (Fig. 5). Observa-se que $94,9 \%$ afirmam ter a necessidade de conter 


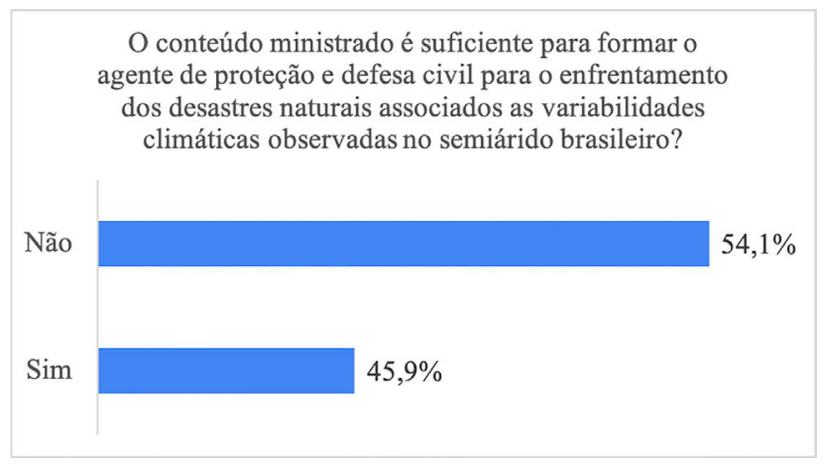

Figura 4 - Expectativa sobre os conteúdos em Meteorologia (Climatologia) ofertados na formação e capacitação continuada dos agentes de proteção e defesa civil da RSANEB. Fonte: Banco de respostas da pesquisa (2020).

nas matrizes dos cursos de formação e nos cursos de capacitação (formação continuada) ofertados as disciplinas que abordem temas associados as variabilidades climáticas da RSANEB. Ressalta-se que, de fato, concordando-se com as reflexões de Cantero (2015) e Giddens (2009), para que haja um planejamento eficaz e eficiente nas ações de defesa civil nessa área, faz-se necessário a compreensão das variáveis responsáveis pela ocorrência de secas e enchentes que são os responsáveis pelos desastres naturais na região.

\section{Conclusões}

Diante das análises e discussões realizadas verificouse que a formação continuada ofertada aos agentes de proteção e defesa civil que atuam na RSANEB necessita passar por um processo de reformulação, incluindo e/ou reforçado a carga horária da ministração de conteúdos sobre os conhecimentos básicos dos fenômenos meteorológicos que modulam o clima e podem causar desastres (secas ou enchentes) na região.

Observou-se que a matriz curricular do curso de formação desses agentes baseia-se na formação para o co- nhecimento da organização estrutural da defesa civil, como conteúdos que vão desde o histórico da implantação da defesa civil no país, a apresentação e prática de preenchimento dos formulários de informações sobre os desastres assim como, na tipificação e codificação dos desastres observados na região.

Em adição, baseado nas informações coletadas, é possível observar que os participantes da pesquisa, agentes de proteção e defesa civil que atam na RSANEB, não se encontram preparados para exercerem plenamente suas funções como prevê a legislação brasileira, ou seja, não estão plenamente preparados para desenvolver um "Conjunto de ações de prevenção, mitigação, preparação, resposta e recuperação destinadas a evitar desastres e minimizar seus impactos sobre a população e a promover o retorno à normalidade social, econômica ou ambiental" (BRASIL, 2016).

Destaca-se o interesse apresentados pelos participantes em ampliar seus conhecimentos referentes as variabilidades climáticas e mudanças no clima da RSANEB que podem influenciar na ocorrência de eventos extremos (secas ou enchentes) na região.

Em geral, os cursos de formação dos agentes de proteção e defesa civil no país e o processo de capacitação (formação continuada) são ofertados por instituições nacionais para todos os estados da federação. Porém, as CEDECs e COMPEDs dos estados e municípios contidos na RSANEB podem assumir a responsabilidade da articulação para oferta de disciplinas nos cursos de formação, de cursos de curta duração, de especializações em nível de pós-graduação, entre outros, para suprir a demanda especifica por formação na área em estudo.

Por fim, menciona-se que dentre os agentes participantes da pesquisa encontra-se $28 \%$ com nível de escolaridade entre fundamental e médio, e que $35,3 \%$ desses possuem curso de graduação, que $46 \%$ dos participantes tem cinco anos ou mais de experiência profissional. Informações que ressaltam a importância da articulação de oferta de formação continuada para os agentes de proteção

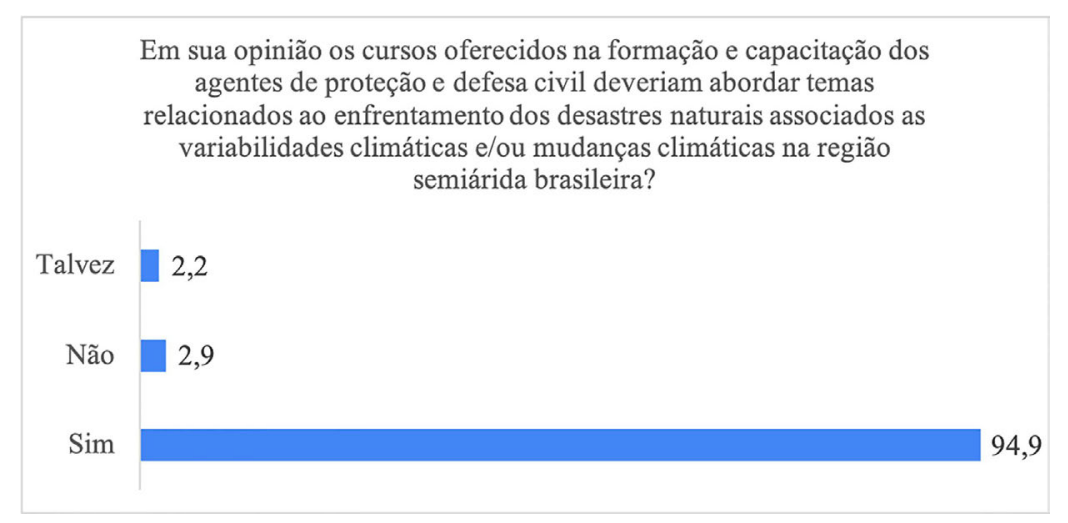

Figura 5 - Expectativa sobre os conteúdos em Meteorologia (Climatologia), ofertados na formação e capacitação continuada dos agentes de proteção e defesa civil da RSANEB. Fonte: Banco de respostas da pesquisa (2020). 
e defesa civil que atuam nas CEDECs e COMPEDs em regiões da RSANEB, para que sejam capazes de compreender a dinâmica dos fenômenos meteorológicos e, com isso, desenvolver planos com ações (preventivas, responsivas e de recuperação) para mitigar os efeitos dos impactos da variabilidade climática observados em suas comunidades.

\section{Referências}

ALVES, J.M.B. Recentes variações climáticas no Nordeste do Brasil com ênfase a precipitação: Relações com o Enos, dipolo de temperatura da superfície do mar no Atlântico Tropical e a oscilação decadal do Pacífico. Revista de Geografia, v. 29, n. 3, p. 147-162, 2012.

BRASIL. Lei ${ }^{\circ}$ 12.608, de 10 de abril de 2012. Institui a Política Nacional de Proteção e Defesa Civil - PNPDEC; dispõe sobre o Sistema Nacional de Proteção e Defesa Civil - SINPDEC e o Conselho Nacional de Proteção e Defesa Civil - CONPDEC; autoriza a criação de sistema de informações e monitoramento de desastres; altera as Leis nos 12.340, de 10 de dezembro de 2010, 10.257, de 10 de julho de 2001, 6.766, de 19 de dezembro de 1979, 8.239, de 4 de outubro de 1991, e 9.394, de 20 de dezembro de 1996; e dá outras providências. Brasília: Diário Oficial da República Federativa do Brasil, 10 abri. 2012.

BRASIL. Instrução Normativa $\mathbf{n}^{\circ} \mathbf{0 2}$, de 20 de dezembro de 2016. Estabelece procedimentos e critérios para a decretação de situação de emergência ou estado de calamidade pública pelos Municípios, Estados e pelo Distrito Federal, e para o reconhecimento federal das situações de anormalidade decretadas pelos entes federativos e dá outras providências. Brasília: Diário Oficial da República Federativa do Brasil, 22 dez. 2016.

BRASIL. Noções Básicas em Proteção e Defesa Civil e em Gestão de Riscos: Livro Base. Brasília: Ministério da Integração Nacional, 2017.

CANTERO, J.G. El Cambio Climático en Europa: Percepción e Impactos 1950-2050. España. Los verdes-ALE/EQUO, 2015.

CARCHEDI, L. Gerenciamento de Desastres. São Paulo. 16 jun. 2006. Disponível em http://resgate.com.br/index.php? op $=$ NEArticle $\&$ sid $=574$. Consultado em 04 de maio de 2020.

CHOU, S.C. Análise de Distúrbios Ondulatórios de Leste Sobre o Oceano Atlântico Equatorial Sul. Dissertação (Mestrado em Meteorologia) - Instituto Nacional de Pesquisas Espaciais (INPE), São José dos Campos, 1990.

DELORS, J. Educação: Um Tesouro a Descobrir. 8. ed. São Paulo: Cortez; Brasília, DF: MEC: UNESCO, 2003.

FACHIN, O. Fundamentos de Metodologia. 3. ed. São Paulo: Saraiva, 2001.

FERREIRA, A.G; MELLO, N.G.S. Principais sistemas atmosféricos atuantes sobre a Região do Nordeste do Brasil e a influência dos oceanos Pacífico e Atlântico no clima da região. Revista Brasileira de Climatologia, v. 1, n. 1, 2005.
FREIRE, P. Pedagogia do Oprimido. 17. ed. Rio de Janeiro: Paz e Terra, 1987.

GIDDENS, A. The Politics of Climate Change. Cambridge: Polity Press, 2009.

GIL, A.C. Métodos e Técnicas de Pesquisa Social. São Paulo: Editora Atlas S.A., 2008.

IPCC. Climate Change 2007: IPCC Fourth Assessment Report, Climate Change 2007: Synthesis Report - Summary for Policymakers, Contribution of Working Groups I-III to the Fourth Assessment Report of the Intergovernmental Panel on Climate Change. BERNSTEIN, L. (ed.). Cambridge: Cambridge University Press, 2007.

KOUSKY, V.E.; GAN, M.A. Upper Tropospheric Cyclone Vortices in the Tropical South Atlantic. Tellus, v. 33, n. 1, p. 538-551, 1981.

MARCONI, M.A.; LAKATOS, E.M. Metodologia do Trabalho Científico: Procedimentos Básicos, Pesquisas Bibliográficas, Projeto e Relatório, Publicações e Trabalhos Científicos. 4. ed. São Paulo: Atlas, 2005.

MARENGO, J.A. Mudanças Climáticas Globais e Seus Efeitos Sobre a Biodiversidade: Caracterização do Clima Atual e Definição das Alterações Climáticas Para o Território Brasileiro ao Longo do Século XXI. Brasília: Ministério do Meio Ambiente, 2006.

Marengo, J.A. Vulnerabilidade, Impactos e adaptação as mudanças de clima no semiárido do Brasil: Parcerias Estrategicas/Centro de Gestão de Estudos Estratégicos. Brasília: Ministério da Ciencia e Tecnologia. Brasília, p. 149-176, 2009.

NÓVOA, A. Formação de Professores e Profissão Docente. Disponível em https://core.ac.uk/download/pdf/12424596. pdf, acesso em: 8 jun. 2020.

RAO, V.B.; HADA, K. Characteristics of rainfall over Brazil: annual variations and connections with the southern oscillation. Theoretical and Applied Climatology, v. 42, n. 1, p. 81-91, 1990.

RASMUSSON, E.M.; CARPENTER, T.H. Variations in Tropical sea surface temperature and surface wind fields associated with the Southern Oscillation/El Niño. Monthly Weather Review, v. 110, p. 354-384, 1982.

SUDENE. Resoluções do Conselho Deliberativo da Sudene de $n^{0} 107$, de $27 / 07 / 2017$ e de $n^{0} 115$, de 23/11/2017. Disponível em: https://www.in.gov.br/web/dou/-/resolucao-n115-de-23-de-novembro-de-2017-739564, acesso em 14 de abril de 2021.

UVO, C.R.B. A Zona de Convergência Intertropical (ZCIT) e Sua Relaçao com a Precipitação da Região Norte do Nordeste Brasileiro. Dissertação de Mestrado em Meteorologia, INPE, São José dos Campos, 1989.

\section{Errata}

O sobrenome do autor Wellington Antonio foi grafado como Brabosa. O correto é Barbosa.

License information: This is an open-access article distributed under the terms of the Creative Commons Attribution License (type CC-BY), which permits unrestricted use, distribution and reproduction in any medium, provided the original article is properly cited. 